\title{
IMPLICIT REPRESENTATIONS OF THE HUMAN INTESTINES FOR SURGERY SIMULATIONS
}

\author{
Laure France $^{1}$, Alexis Angelidis ${ }^{2}$, Philippe Meseure ${ }^{1}$, Marie-Paule Cani ${ }^{2}$, \\ Julien Lenoir ${ }^{1}$, Francois Faure ${ }^{2}$ and Christophe Chaillou ${ }^{1}$
}

\begin{abstract}
In this paper, we propose a modeling of the intestines by implicit surfaces for abdominal surgery simulation. The difficulty of such a simulation comes from the animation of the intestines. As a matter of fact, the intestines are a very long tube that is not isotropically elastic, and that bends over itself at various spots, creating multiple self-contacts.

We use a multiple component model for the intestines: The first component is a mechanical model of their axis; the second component is a specific sphere-based model to manage collisions and selfcollisions; and the third component is a skinning model to define their volume. This paper focuses on the better representation for skinning the intestines. We compare two implicit models: Surfaces defined by point-skeletons and convolution surfaces.

A direct application of this simulation is the training of a typical surgical gesture to move apart the intestines in order to reach certain areas of the abdomen.

Keywords: Surgical simulation, virtual reality, implicit surfaces, real-time computation, application to medical domain.

Résumé. Dans cet article, nous proposons une modélisation des intestins par surfaces implicites pour la simulation de chirurgie abdominale. La difficulté d'une telle simulation provient de l'animation des intestins. En effet, les intestins sont un très long tube qui n'est pas élastique de manière isotrope, et qui se replie sur lui-même en de nombreux endroits, créant de multiples auto-contacts.

Nous utilisons un modèle à plusieurs composants pour les intestins : le premier composant est un modèle mécanique réduit à leur axe ; le deuxième composant est un modèle spécifique basé sur des sphères pour gérer les collisions et auto-collisions ; et le troisième composant est un modèle d'habillage pour définir leur volume. Cet article se focalise sur la meilleure représentation pour habiller les intestins. Nous comparons deux modèles implicites : les surfaces définies par des squelettes ponctuels et les surfaces de convolution.

Une application directe de cette simulation est l'apprentissage d'un geste chirurgical caractéristique pour rassembler les intestins afin de dégager certaines zones de l'abdomen.

Mots clés: Simulation chirurgicale, réalité virtuelle, surfaces implicites, calculs temps-réel, application au domaine médical.
\end{abstract}

\footnotetext{
${ }^{1}$ LIFL, Université Lille I, Villeneuve d'Ascq.

[Laure.France|Philippe.Meseure|Julien.Lenoir|Christophe.Chaillou]@lifl.fr

2 iMAGIS-GRAVIR, INRIA Rhône-Alpes, Montbonnot.

[Alexis.Angelidis|Marie-Paule.Cani|Francois.Faure]@imag.fr
} 


\section{INTRODUCTION}

Laparoscopic surgery is a surgical technique that uses a camera and surgical tools inserted into the body via small incisions. This technique leads to unusual working conditions that require many rehearsals. The training then could be performed on a simulator. Surgeons would learn the surgical gestures on physically based virtual organs that react to their actions.

Some endoscopic simulators exist, usually consisting in simulating the interaction of surgical tools with a single organ. Dynamic virtual organs have already been modeled, such as the liver $[5,10,12,16]$ or the colon [14]. Nevertheless, to our knowledge, there are no current satisfying solutions for the viscera, composed of very deformable objects that are in contact in various spots and may undergo very large displacements.

The goal of our simulator is to clear an area to treat, by pushing/pulling and maintaining the intestines in another place of the abdominal cavity. Indeed, this clearing stage is needed before surgeries such as the gallbladder removal or colon tumors. However, this action rises several challenges: Large displacements, contacts and self-collisions.

We developed a first model of the intestines in [13], composed of three components: A mechanical model to define their physical behavior; a model for self-collisions between their parts and collisions with other objects; and a skinning model to represent and display their shape. However, the skinning model based on parametric generalized cylinders was not satisfying, since it could result in surface discontinuities in highly curved regions and in volume loss.

In this paper, we address this problem by using implicit surfaces, known for representing quite naturally organic shapes [2]. Indeed, they follow a skeleton in an intuitive way regardless of its deformation. Moreover, this representation allows the generation of contact between surfaces [6]. Lately, implicit surfaces were even adapted to levels of detail (LOD) by taking into account multi-resolution aspects [1].

The paper is organized as follows: Section 2 reviews our three component model for the intestines. Sections 3 and 4 present the two alternative skinning models and discuss their ability to model the intestines. Section 5 concludes and discusses some future work.

\section{A three COMponent MOdel For the intestines}

We want to simulate the evolution of the behavior and the visual aspect of the intestines under the action of surgical tools, and to return an appropriate haptic feedback via the user interface. To simulate the physical behavior of the intestines, we use a mechanical model that animates their virtual skeleton. The intestines skeleton can be represented as a curve with mechanical properties. Next, since the intestines may undergo self-collisions and collisions with their environment and some surgical tools, we use a detection model based on the mechanical model to compute these collisions and their response. Finally, we provide a skinning model in order to represent and display the shape of the intestines (but with no mechanics at this level). We briefly present our first model (further details can be found in [13]) and discuss the flaws of the parametric skinning model we used.

\subsection{Mechanical Model}

We use a cubic Catmull-Rom [9] segmented spline to define the intestines axis (their skeleton). This definition allows the user to control the shape of the spline just by modifying the control points. In order to animate the spline, we use a Lagrangian formalism inspired from [17]. This formalism takes into account the continuity of the object and thus enables a continuous mass distribution along the curve. Moreover, it allows external actions and/or constraints to occur anywhere along the spline.

The formulation of the equations leads to the resolution of a system of the form $\mathcal{M A}=\mathbf{B}$, namely the resolution of $\mathbf{A}=\mathcal{M}^{-1} \mathbf{B}$, with $\mathcal{M}$ the generalized mass matrix, $A$ the acceleration of the degrees of freedom (the control points), and $B$ the forces. It was pointed out that $\mathcal{M}$ is a diagonal block matrix with identical diagonal blocks $M . M$ is a symmetric, time-independent matrix, allowing the pre-computation of the inverse matrix, yielding in a faster resolution of the equation system. Besides, we take benefit of the locality of the 
Catmull-Rom spline: For any cubic spline, the matrix $M$ is band. Finally, the system resolution complexity becomes $O(n)$. Once this system solved, we get the acceleration of the degrees of freedom. We use a RungeKutta integration scheme to estimate the new velocities and new positions of the control points.

\subsection{Collision/Self-collision Model}

Our collision detection method uses an approximation of the object shape by bounding spheres. A 3D grid that discretizes the space is used to speed up the process: Collisions only need to be performed between spheres belonging to a same voxel. This process is iterated for each sphere added to the grid. Since the penalty methods are well suited to deformable objects, they are used for collision response (see [11] for more details).

In the case of the intestines, it consists of placing spheres of the same radius along their skeleton curve, uniformly according to the parametric abscissa (see Figure 1). When a surgical tool interacts with the intestines, the intestines spheres ensure the detection of the collision and provide penalty forces as reaction response, yielding in the movement and/or deformation of the intestines and in forces returned by the feedback device.

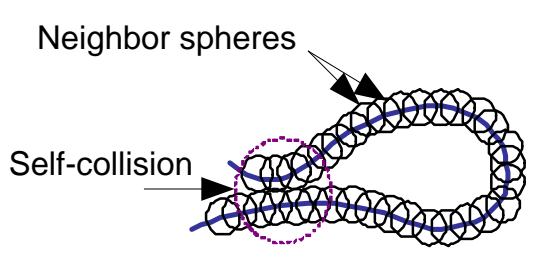

FIGURE 1. Sphere distribution along the intestines axis.

Self-collisions between intestines folds are managed by using the same method. Nevertheless, in the detected collisions, we must discard the spheres that are neighbors along the intestines axis (see Figure 1).

\subsection{Skinning Model}

The mechanical model runs at $100 \mathrm{~Hz}$ or more, independently from the skinning model, displayed at $25 \mathrm{~Hz}$. At each display step (that may be larger than the simulation step), a skinning of the intestines must be generated. It must depend upon the position and the placement of their skeleton. In other words, the skeleton describing the general motion of the object, the skinning has to follow it, providing the resulting shape of the object.

Different methods exist to define a volume, some of them are more adapted to a tubular volume based on a linear skeleton. The idea is to create a volume delimited by a surface around the skeleton. In our previous model [13], we defined the intestines surface as a parametric surface around the discrete skeleton based on the spline points, at a distance representing the intestines radius. This representation allowed an interactive display. Nevertheless, it suffers from two major drawbacks: First, the resulting shape is imperfect for high curvatures, and suffers from tangent and volume discontinuities; second, there is no direct method to deform it in order to model exact contact between surfaces.

On the other hand, implicit surfaces particularly suit organic shapes, since they control a volume around the skeleton, enabling realistic curved shapes and geometric contact between surfaces [7]. The remainder of the article presents two methods based on implicit surfaces for the display of the intestines.

\section{Implicit Intestines Using Point-Skeletons}

\subsection{Method}

An implicit surface is defined by the set of points such that the value of a given field function $f$ for these points $P$ equals to a given value $e$, namely the iso-value, for which the iso-surface is drawn: $f(P)=e$. The evaluation of the field function for any point allows a straightforward detection of the interior/exterior of the object. This checking simplifies the collision detection between objects, and thus the blending control. 
One possibility to generate an implicit surface from a skeleton is to use distance surfaces [4]. Distance surfaces compute the field value at a given point $P$ from its distance to its closest point on the skeleton. The blending of several skeletons contributions is directly performed by summing their field values. This technique then suppresses surface folds at the joint of skeletons, but may create bulges [3].

We define the intestines surface as an implicit surface generated by discrete point-skeletons positioned along the spline. The strength of their field value is tuned according to the radius of the intestines (see Figure 2 (a)). During the animation, the skeleton shape varies as the spline points move. However, if two points of the elastic spline are too far from each other, there is no more blending between them, thus separating the object into two parts. In order to avoid these topology changes, the number of sampling points must change during the simulation. This is done by adaptively placing spheres along the curve at intervals which range is a function of the spline segment length and the radius of the spheres. Next, we use a real-time implementation of the marching cubes algorithm proposed by [19] for the visualization of the implicit surface.

\subsection{Results}

The blending property of the implicit surface ensures a continuous shape for the intestines model (see Figure 2 (b)). However, it is particularly difficult to provide a constant radius. Indeed, the variation of the number of skeletons leads to fluctuations of the geometry (this could represent the spasms of the intestines, but we want to control them). Moreover, care must be taken to avoid blending between non-consecutive parts. It requires the use of the blending graph. Unfortunately, in our implementation, the blending control cannot be done at a sufficient rate due to the marching cube method to display the surface.

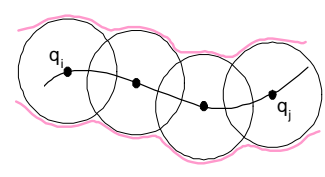

(a) Model.

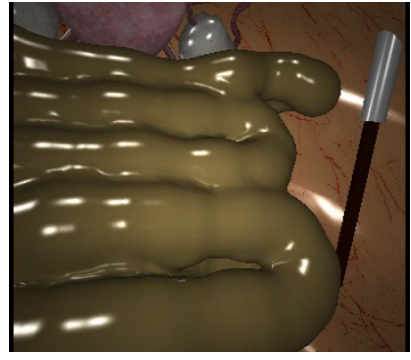

(b) Interactive display.

FIGURE 2. Implicit intestines using point-skeletons.

\section{Implicit Intestines generated by a Convolution Surface}

\subsection{Method}

In order to avoid the flaws of a varying number of point-skeletons, we must use the whole skeleton curve or at least a linear approximation of this curve to generate the intestines geometry. One possibility is to use convolution surfaces [3], and to represent the shape as a set of connected convolution segment-skeletons. This representation avoids bulges on the surface that coats the segments, and allows the number of segments to change at each time step without creating jumps on the implicit surface geometry, as long as newly introduced vertices are smoothly translated.

For a single convolution segment-skeleton, the field value at a point $P$ is the sum of the contribution of all the point-skeletons along the segment. This integral has a closed-form solution for various point-skeleton kernel functions $[8,15,18]$. The solution proposed in [8] is the fastest, and its expression is: $f(P)=\frac{\sin \alpha_{1}-\sin \alpha_{2}}{d^{2}(P, H)}$, with 
$d(P, H)$ the distance between $P$ and its projection $H$ on the skeleton segment, $\alpha_{1}$ and $\alpha_{2}$ the signed angles between this axis of projection and the lines linking $P$ to the extremities of the skeleton segment.

A straightforward way of modeling a smooth complex surface would then consist in summing all the individual field contributions of the simple segment-skeletons. This would not however prevent the surface from blending between non-consecutive parts. Therefore, we use the controlled blending technique developed in [1], that first needs to describe the sampling of the implicit surface.

To display the surfaces at interactive rate, we use the seed-based method of [8] which takes benefit from the temporal coherence. Seeds migrate towards the surface according to the iso-surface. Initially, they start from the skeleton, and in the remainder of the simulation, they start from their previous positions. The discretization of the surface triangulation is adaptive, allowing the surface to be rendered at different levels of detail. To produce the adaptive sampling, a rough polygonization is initially attached to a few sampling points, and then refined recursively by a uniform subdivision until a certain criterion is satisfied.

Finally, the unwanted blending is managed by local convolution [1] (see Figure 3 (a)). The display method assigns to every point $P_{i} \in \mathbb{R}^{3}$ on the surface a parameter $u_{i}$ along the skeleton, which corresponds to the attached point of the seed axis. A local portion of the skeleton around $u_{i}$ is used to compute $f\left(P_{i}\right)$, which is a neighborhood of constant size $D$ around $u_{i}$. The field value is then computed exactly as before, with the exception that only the useful part of the skeleton is considered in this case. A constrain on $D$ is $D<2 R$ ( $R$ is the intestines radius), since two points on the surface separated by a distance greater than $2 R$ must not influence each other.

\subsection{Results}

With this model, the visual rendering of the intestines is satisfying: There is no blending, and no bulges created (see Figure 3 (b)). However, the computation time is still too slow if we want a fine discretization of the surface object.
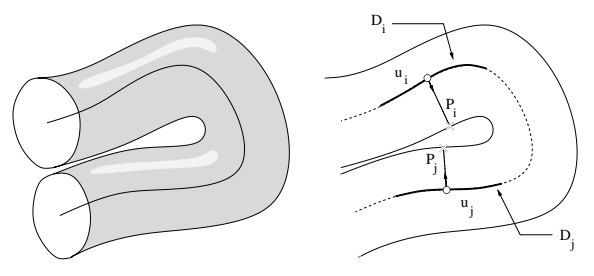

(a) Model.
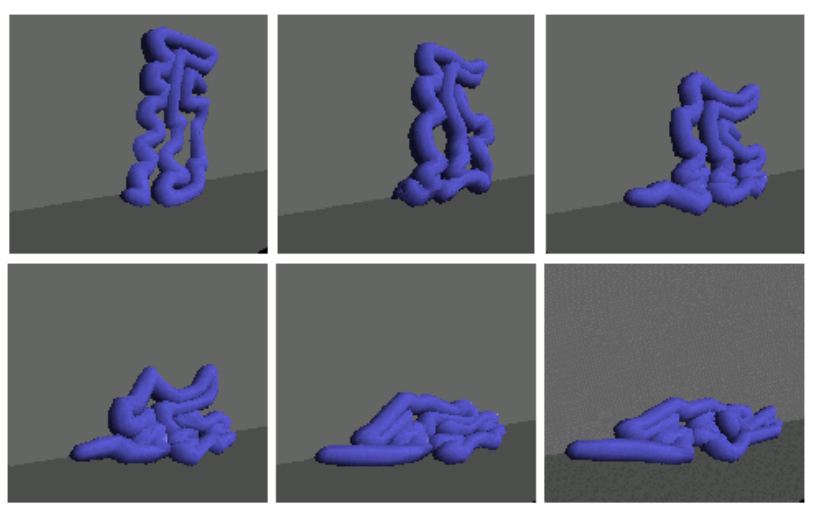

(b) Display of intestines only animated with gravity.

FIGURE 3. Implicit intestines generated by a subdivision curve.

\section{Conclusion}

In this paper, we presented a method to simulate at interactive rate the intestines in the abdominal cavity, for surgical training purposes. The intestines are very deformable objects, and during a surgery, they may undergo large displacements and multiple interactions. The intestines model is based on a skeleton defined by 
a spline to compute its mechanical motion from the Lagrange formalism. Collision spheres are then used for a fast collision detection, providing real-time feedback via the haptic device. The paper focused on the skinning of this model. It compared two implicit solutions that offer quite good results in terms of visual effects concerning the movements and deformations of the intestines. We showed that this skinning by implicit surfaces may be attractive in the context of a surgical simulator. The introduction of adaptive implicit surfaces based on convolution is interesting since the animation could be displayed at different levels of detail to obtain interactive rate whatever the workstation.

Currently, only the discretization of the surface triangulation is adaptive. A future work could consist in dynamically adapting the discretization of the skeleton according to the varying curvature. It could be interesting to deal with both aspects in order to tune the parameters to obtain computations and display in real-time. Moreover, it could be possible to add the contact surfaces presented in [1] so that the contact is better handled. On the other hand, self-collisions could be detected more precisely by taking into account the information provided by the implicit surface.

\section{ACKNOWLEDGMENTS}

This work is supported by INRIA (French National Institute for Research in Computer Science and Control) as part of the ARC SCI (research action for Intestines Surgery Simulator). The authors would like to thank people of IRCAD (French Digestive Cancer Research Institute) for their constructive discussions.

\section{REFERENCES}

[1] A. Angelidis, P. Jepp, and M.-P. Cani. Implicit modeling with skeleton curves: Controlled blending in contact situations. Shape Modeling International, 2002.

[2] J. Bloomenthal, C. Bajaj, J. Blinn, M.-P. Cani, A. Rockwood, B. Wyvill, and G. Wyvill. Introduction to Implicit Surfaces. Morgan Kaufmann, July 1997.

[3] J. Bloomenthal and K. Shoemake. Convolution surfaces. Computer Graphics, 25(2):251-256, July 1991.

[4] J. Bloomenthal and B. Wyvill. Interactive techniques for implicit modeling. Computer Graphics, 24(2):109-116, March 1990.

[5] M. Bro-Nielsen and S. Cotin. Real-time volumetric deformable models for surgery simulation using finite elements and condensation. Computer Graphics Forum 15(3):57-66, 1996.

[6] M.-P. Cani. An implicit formulation for precise contact modeling between flexible solids. In Computer Graphics, pages 313-320. ACM, aug 1993. Published under the name M.-P. Gascuel.

[7] M.-P. Cani and M. Desbrun. Animation of deformable models using implicit surfaces. IEEE Transactions on Visualization and Computer Graphics, 1(3), March 1997. Published under the name M.-P. Cani-Gascuel.

[8] M.-P. Cani and S. Hornus. Subdivision curve primitives: a new solution for interactive implicit modeling. In Shape Modelling International, May 2001.

[9] R. Catmull and R. Rom. A class of local interpolating splines. Computer Aided Geometric Design, Academic Press, 1974.

[10] S. Cotin, H. Delingette, and N. Ayache. Real time volumetric deformable models for surgery simulation. Visualization in Biomedical Computing, volume 1131. Springer Verlag, 1996.

[11] J. Davanne, P. Meseure, and C. Chaillou. Stable haptic interaction in a dynamic virtual environment. IEEE/RSJ International Conference on Intelligent Robots and Systems, 2002.

[12] G. Debunne, M. Desbrun, M.-P. Cani, and A.H. Barr. Dynamic real-time deformations using space and time adaptive sampling. Computer Graphics, August 2001.

[13] L. France, J. Lenoir, P. Meseure, and C. Chaillou. Simulation of a minimally invasive surgery of intestines. Virtual Reality International Conference, pp 21-27, 2002.

[14] A. Haristis, D. Gillies, and C. Williams. Realistic generation and real time animation of images of the human colon. Computer Graphics Forum 1992, vol. II, 3:367-379, 1992.

[15] S. Hornus, A. Angelidis, and M.-P. Cani. Implicit modelling using subdivision-curves. The Visual Computer, 2002.

[16] G. Picinbono, H. Delingette, and N. Ayache. Non-linear and anisotropic elastic soft tissue models for medical simulation. IEEE International Conference Robotics and Automation, May 2001.

[17] Y. Remion, J.M. Nourrit, and D. Gillard. Dynamic animation of spline like objects. WSCG, 1999.

[18] A. Sherstyuk. Kernel functions in convolution surfaces: a comparative analysis. The Visual Computer, 15(4), 1999.

[19] F. Triquet, P. Meseure, and C. Chaillou. Fast polygonization of implicit surfaces. WSCG (2):283-290, February 2001. 